\title{
Principios para la elaboración del Atlas de incidencia delictiva de las principales ciudades de Quintana Roo
}

DOI: 10.22403/UQROOMX/TYP06/05

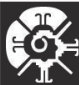

RESUMEN
Thomas Ihl,* Oscar Frausto, ** Javier Tun Chim, *** Celina Izquierdo***** Manfred Rolfes******

La violencia y delincuencia son temas emergentes y urgentes en México. En los últimos años, la incidencia delictiva juega un papel importante en la percepción pública. Hay señales claras de que el número de delitos no denunciados aumenta más rápido en contraste con los delitos registrados. El presente artículo trata los principios teóricos y metodólogicos para la elaboración del atlas del delito de Quintana Roo, el cual se basa en la experiencia acumulada por el Observatorio Urbano de la Riviera Maya en los últimos años.

\section{Palabras} CLAVE

Quintana Roo, atlas, incidencia delictiva, mapeo del delito.

\footnotetext{
*Universidad de Quintana Roo, Observatorio Urbano de la Riviera Maya / Universidät Postdam / thomas@uqroo.mx

** Universidad de Quintana Roo, División de Desarrollo Sustentable / Universidad BauhausWeimar / ofrausto@uqroo.mx

***Universidad de Quintana Roo, Observatorio Urbano de la Riviera Maya / javtun@uqroo.mx *****niversidad del Caribe, Observatorio Urbano de Violencia Social y Género de Benito Juárez / cizquierdos@unicaribe.edu.mx ******Universität Postdam / mrolfes@uni-postam.de
} 


\section{Introducción}

Un atlas es una colección de mapas o documentos cartografiados que se preparan especialmente para brindar una información global o lo más completa posible sobre los fenómenos geográficos en un espacio determinado (Moellering, Aalders y Crane, 2005). Su importancia es indiscutible para la enseñanza y para la planificación del desarrollo de un país o una región. Por ejemplo, el Atlas nacional de México (IG-UNAM, 1992) presenta la estructura de los componentes medioambientales, sociales, humanos y económicos del país.

Un atlas es una herramienta para tomar decisiones; compila, muestra y señala dónde, cómo y qué sobre un fenómeno específico. Hoy en día, mediante los sistemas de información geográfica (SIG), diseñados en particular para el monitoreo y el control de la incidencia delictiva, se plantea fortalecer la prevención de la delincuencia en las ciudades de Cancún y Cozumel, Quintana Roo, como modelo que fundamenta el programa federal de Rescate de Espacios Públicos.

\section{Generalidades}

Durante los últimos 20 años, en muchas de las grandes ciudades del mundo el crecimiento de la delincuencia se ha convertido en un serio problema. En los países del Norte, la pequeña delincuencia aumentó entre tres y cinco por ciento anual de la década de los setenta a la de los noventa. A partir de esta última, la tasa de criminalidad urbana ha empezado a estabilizarse, con excepción de la criminalidad de los jóvenes (I 2-25 años), la cual se ha vuelto cada vez más violenta, además de que la edad de ingreso a esta actividad ha disminuido de los 15 a los 12 años (ONU-Hábitat, 2006).

Desde de los años ochenta, la criminalidad ha crecido de manera exponencial en los países del Sur. Fenómenos como los niños de la calle, el abandono escolar, el analfabetismo, la exclusión social masiva, las repercusiones de las guerras civiles y el comercio ilegal de armas ligeras han acentuado dicho proceso. Este aumento de la criminalidad se desarrolla en un contexto caracterizado tanto por el mayor tráfico y abuso de drogas, como por su coexistencia con la globalización de la criminalidad organizada, la cual contribuye a desestabilizar regímenes políticos y a incrementar los efectos de las crisis económicas, entre los cuales se destaca la incorporación de los jóvenes a las actividades delictivas en calidad de mano de obra de bajo costo. 
"Las causas de la delincuencia son múltiples. Las investigaciones han mostrado que no existe una causa única sino una serie de causas interrelacionadas" (ONU-Hábitat, 2006). Por tanto, se pueden establecer tres principales categorías de ellas: las sociales, las institucionales y las referentes al entorno urbano y físico.

I. Las situaciones de exclusión social debidas al desempleo, a la marginación prolongada, al abandono escolar o al analfabetismo y a las modificaciones estructurales de la familia se encuentran con frecuencia entre las causas sociales de la delincuencia. Contrario a la creencia difundida, la pobreza no constituye una fuente directa de la delincuencia.

2. Entre las causas institucionales, la principal es la inadecuación del sistema de justicia penal (policía, justicia y cárceles) a la delincuencia urbana y a su crecimiento. Por otra parte, la ineficiencia de la justicia y la impunidad frente a delitos como el lavado de dinero, el crimen organizado, la participación en actividades mafiosas, la corrupción y la violación de derechos humanos favorecen los comportamientos delictivos y la arbitrariedad.

3. Entre las razones ligadas al entorno, están la urbanización descontrolada, la carencia de servicios urbanos, la ausencia del concepto de seguridad en las políticas urbanas, el surgimiento masivo de espacios semipúblicos (malls, estaciones, etc.) y la ilegalidad que se instala en los barrios transformados en zonas bajo el control de pequeñas mafias locales.

De los resultados del reporte ONU-Hábitat (2006) se desprende una primera consecuencia: la percepción de inseguridad generalizada, que cristaliza el conjunto de miedos de la población (inseguridad frente al empleo, al porvenir de los hijos, a la violencia intrafamiliar, etc.). La segunda implicación es el efecto de la inseguridad en los sectores pobres. Las investigaciones muestran que la violencia urbana daña mayormente a los sectores pobres porque tienen pocos medios para defenderse de ella y, sobre todo, porque la inseguridad quiebra su capital social e impide su movilidad. La tercera secuela es el aumento de los costos de la seguridad, que alcanzan entre cinco y seis por ciento del producto interno bruto en los países del Norte y entre el ocho y el diez por ciento en los del Sur. Una cuarta consecuencia es el desarrollo masivo de las empresas privadas de seguridad, las cuales, durante los últimos años del siglo $\mathrm{XX}$, alcanzaron un crecimiento anual de 30 por ciento en los países del Sur y de ocho por ciento en los del Norte.Y, por último, están los ensayos de respuesta 
pública a esta nueva situación de creciente criminalidad caracterizados por dos tendencias globales: por una parte, las tentativas de gobiernos por reforzar la seguridad sólo mediante el uso de la represión y, por la otra, aquellas que privilegian la prevención al mismo tiempo que la represión.

\section{Fundamentos del Atlas de incidencia delictiva}

El tema del delito ha sido estudiado primordialmente por las ciencias sociales; por el derecho, el cual se ocupa del funcionamiento del sistema judicial y de la ejecución penal, y por la sociología, que trata de desentrañar la vinculación de los hechos delictivos con la marginación social, la violencia policial, la migración, etcétera (Igarzábal de Nistal, 2006).

Desde la perspectiva de la distribución espacial de los delitos, Pain (2000) sugiere que la contribución de la geografía al estudio de la violencia está en "situarla", caracterizando el espacio en donde se produce, los efectos respecto a la cohesión social de las ciudades y las implicaciones en la definición morfológica de aquéllas. Entre las líneas de trabajo que se siguen en el campo de la geografía del delito destacan: a) el análisis de la influencia de las características de los individuos con relación a la percepción de la seguridad, b) el nivel de riesgo que han de asumir los habitantes, c) el uso del espacio, d) el alcance de la delincuencia y e) la seguridad en cuanto a la percepción de la calidad de vida (Lahosa, 2002).

\section{Mapeo del delito}

Para elaborar el Atlas de incidencia delictiva se desarrollaron las siguientes actividades:

a) Se definió el marco conceptual de incidencia delictiva, con el fin de dar soporte a la estructura y funcionalidad del Atlas. Para ello se tomaron en consideración los principios de la elaboración cartográfica temática del mapeo del delito de Boba (2005), Chainey y Ratcliffe (2005), y Osborne y Wernicke (2003). Para el análisis de los datos mediante el SIG, se siguieron los principios de Eck et al. (2007).

b) Se compiló información oficial sobre el delito, para lo cual se obtuvieron las bases de datos del Instituto Nacional de Estadística, Geografía e Informática (INEGI, 2005a, b y c), de la Procuraduría General de la República (PGR, 2005) 
y del Instituto Ciudadano de Estudios Sobre la Inseguridad (ICESI, 2002), para construir y examinar el diagnóstico base de la incidencia delictiva en Quintana Roo.

c) Con fundamento en el análisis de la información oficial, y considerando que en estas cifras sólo se incluyen los delitos denunciados, se estructuró una encuesta de base con el propósito de conseguir información directa por medio de una muestra representativa en las ciudades de Cancún y Cozumel. Las encuestas se aplicaron durante julio y agosto de 2007 y tuvieron como objetivos: evaluar la cifra negra y la cultura de la denuncia, estimar los hechos delictivos denunciados y no denunciados, conocer la percepción sobre la inseguridad y la evaluación de la seguridad, identificar el tipo de actividades cotidianas inhibidas por miedo a ser víctima de conductas delictivas y tipificar a los delincuentes.

d) Se construyó el sistema de información de la incidencia delictiva de las principales ciudades de Quintana Roo (Cozumel y Cancún) con objeto de mapear la información oficial y la obtenida en la encuesta. Para ello se utilizó el sistema de soporte ArcGIS 9.2 y el programa Crimestat III (Levine, 2007).

e) Finalmente, se construyó el portal de Internet del Atlas de incidencia delictiva de las principales ciudades del estado de Quintana Roo, el cual se integra en seis apartados:

I. Inicio o marco de referencia sobre los delitos e incidencia delictiva.

2. Programas: que concierne al vínculo con los usuarios del Atlas, principalmente el monitoreo que se lleva a cabo dentro de la organización Hábitat de las Naciones Unidas mediante la acción Ciudades Seguras y el programa gubernamental federal de la Secretaría de Desarrollo Social (Sedesol) Rescate de Espacios Públicos.

3. Metodología: en el cual se describe a detalle el proceso de compilación, revisión y mapeo de los indicadores sobre incidencia delictiva por ciudad.

4. Mapas: es el portal interactivo del Atlas y está diseñado para los usuarios directos (Procuraduría, municipios, organizaciones civiles, académicos y público en general); asimismo, el programa utilizado para acceder a la información es de fácil ejecución (se emplea el programa MapViewSVG 4.2). 
Principios para la elaboración

del Atlas de incidencia delictiva

5. Proyectos: donde se muestra la sinergia que ha permitido el proyecto financiado por el Fondo Mixto del Consejo Nacional de Ciencia y Tecnología-Quintana Roo (Conacyt-QR), así como el perfil de los participantes del proyecto y sus últimas publicaciones relacionadas con el Atlas.

6. Vínculos: que están ligados al tema de incidencia delictiva y a las organizaciones que lo tratan (figura I).

\section{Consideraciones finales}

El diseño del Atlas de incidencia delictiva obedece a una necesidad de identificación, control y vigilancia de la prevención de la delincuencia en un territorio específico y conforme a una determinada organización espacial.

Con el fin de desarrollar una base de datos sobre los delitos, se definió tanto el marco de acción legal (delitos reconocidos y castigados por la ley), como una organización de la información oficial (INEGI, ICESI, Procuraduría General de Justicia) y no oficial (encuestas de opinión y registros de diarios y noticieros).

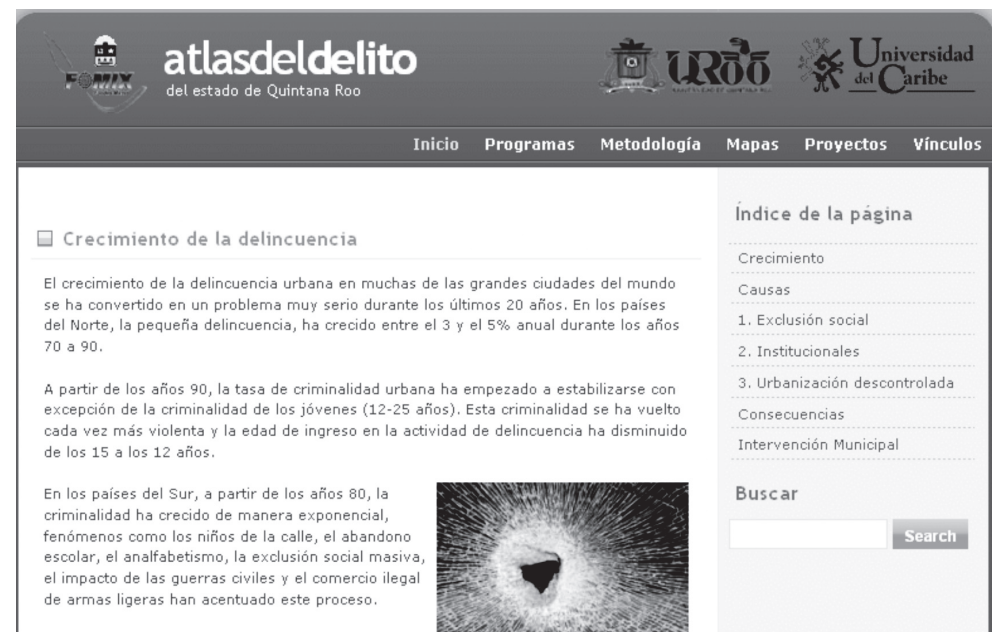

Figura I. Portal de INTERNet del AtLAS DE INCIDENCIA Delictiva de las PRINCIPALES CIUDADES DEL ESTADO DE QUINTANA Roo <www.atlasdeldelito.info> 
Ihl, Frausto,Tun, Izquierdo, Rolfes



\section{Levenda:}

\section{Delitos denunciados (2006)}

- Delito denunciado Traza urbana

\section{Densidad}

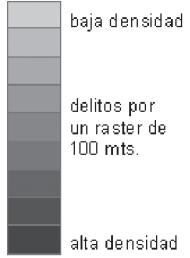

De 1,866 delitos denunciados en el año 2006 son:



口 localizados

口no localizados

$\square \sin$ nombre de calle
0 $250 \quad 500 \quad 1000 \mathrm{~m}$

Proyección: UTM WGS 84 Zona 16N

Fuentes: - INEGI (Traza urbana)

- Delitos denunciados de

Procuraduría general de Justicia, 2006

Autores: Thomas |hl

Observatorio Urbano Riviera Maya 徨灌

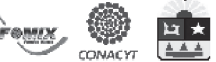

Figura 2. Delitos denunciados y su densidad en la ciudad de Cozumel, Quintana Roo 
Principios para la elaboración del Atlas de incidencia delictiva



\section{Levenda:}

\section{Delitos denunciados (2006)}

- Delito denunciado Traza urbana

\section{Densidad}

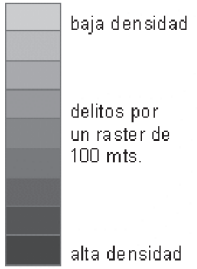

De 1,866 delitos denunciados en el año 2006 son:

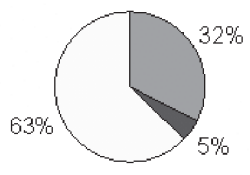

o $250 \quad 500 \quad 1000 m$

Proyección: UTM WGS 84 Zona 16N

Fuentes: - INEGI (Traza urbana)

- Delitos denunciados de Procuraduría general de Justicia, 2006

uno localizados

Autores:

Thomas Ihl

Observatorio Urbano Riviera Maya

$\square$ sin nombre de ronsy CONACYT

Figura 3. Percepción de la inseguridad en la ciudad de Cozumel, Quintana Roo 
Así, considerando el marco conceptual y la base de datos, se conformó el Atlas de incidencia delictiva de las principales ciudades del estado de Quintana Roo, cuyo eje rector es la representación cartográfica del fenómeno de la delincuencia en las ciudades de Cancún, Playa del Carmen y Cozumel. En las figuras 2 y 3 se muestran los modelos cartográficos del Atlas.

Desde la perspectiva de la aplicación del Atlas de incidencia delictiva a las políticas públicas, y con objeto de mejorar la percepción de la población en torno a la seguridad, éste se orienta a modificar las variables que inciden en el desarrollo del delito, para lo cual es necesario identificar los lugares más vulnerables.

La Sedesol, por medio del Programa Rescate de Espacios Públicos, es la principal usuaria del modelo del Atlas, pues el objetivo del programa es fomentar la identidad comunitaria, la cohesión social y la generación e igualdad de oportunidades, así como contribuir a la disminución de la pobreza urbana, prevenir conductas antisociales y mejorar la calidad de vida. Está dirigido a dignificar áreas marginadas y espacios comunitarios, influyendo positivamente en aquellos problemas sociales que afectan la seguridad de las comunidades, mediante la protección, la conservación y el aprovechamiento de los espacios públicos de las ciudades, entendidos como todas aquellas áreas de uso común: plazas, alamedas, áreas verdes, parques, jardines, espacios deportivos y culturales, camellones, centros de ciudad, bosques y lagos, entre otros.

Por su parte, el visualizador de los delitos (mapas interactivos a través del portal de Internet) ha resultado una herramienta de gestión para los ministerios públicos municipal y estatal, pues ayuda a dar un seguimiento sistemático de las causas en cuanto a dónde, cuándo y con qué frecuencia ocurren los delitos, presentando tendencias de localización de sus diversos tipos. El empleo conjunto del visualizador y de las encuestas de percepción de inseguridad contribuye a orientar acciones de seguridad pública.

\section{Agradecimientos}

Se agradece al Fondo Mixto Conacyt-Quintana Roo por el apoyo financiero al Proyecto Fortalecimiento de la Seguridad Pública de las Principales Ciudades en Q. Roo a través de la Generación, Manejo y Uso del Atlas de incidencia delictiva (Clave: QROO-2006COI-55797); a la Universidad de Quintana Roo, campus Cozumel; a la Universidad del Caribe; al Observatorio Urbano de Violencia Social y Género de Benito Juárez; y al Observatorio Urbano de la Riviera Maya, por el complemento financiero para desarrollar el Atlas. 


\section{FUENTES CONSULTADAS}

Boba, R. (2005). Crime Analysis and Crime Mapping. Thousand Oaks: Sage Publications, $30 \mathrm{I} \mathrm{p}$.

Chainey, Spencer y Jerry Ratcliffe (2005). GIS and Crime Mapping. Mastering GIS:Technology,Applications \& Management. Chichester:John Wiley \& Sons, $448 \mathrm{p}$.

Eck, J. et al. (2007). Mapping Crime: Understanding Hot Spots. Washington: U.S. Department of Justice, Office of Justice Programs, National Institute of Justice.

ICESI (2002). "La percepción de inseguridad en las principales regiones urbanas de México", Gaceta 6 [en línea]. Instituto Ciudadano de Estudios Sobre la Inseguridad. Disponible en:www.icesi.org.mx/documentos/ publicaciones/gacetas/percepcion_inseguridad_principales_regiones de_mexico.pdf

IG-UNAM ( 1992). Atlas nacional de México, ts. I, „I y III. México: Instituto de GeografíaUniversidad Nacional Autónoma de México.

lgarzábal de Nistal, M.A. (2006).“Enfoque espacial de la problemática del delito aplicado a la Ciudad Autónoma de Buenos Aires”, en Fraile, P. et al. Paisaje ciudadano, delito y percepción de la inseguridad. Investigación interdisciplinaria del medio urbano, 29-52.

INEGI (2002) Estadísticas judiciales en materia penal, Cuaderno núm. I0 [en línea]. Disponible en: www.inegi.gob.mx [2007, I 5 de diciembre]

_ (2005a). Estadísticas judiciales en materia penal de los Estados Unidos Mexicanos 2005. México: Instituto Nacional de Estadística, Geografía e Informática.

_ (2005b). Tercera Encuesta Nacional sobre Inseguridad ENSI, 2005 [Base de datos]. México: INEGI.

(2005c). Estadísticas judiciales en materia penal. México: INEGI (Serie Boletín de estadísticas continuas, demográficas y sociales), $38 \mathrm{p}$.

Lahosa, Josep M. (2002). “Delincuencia y ciudad. Hacia una reflexión geográfica comprometida”. Biblio 3W. Revista Bibliográfica de Geografia y Ciencias Sociales, VII (349), 20 de febrero [en línea]. Universidad de Barcelona. Disponible en: www.ub.es/geocrit/b3w-349.htm

Levine, Ned (2007). CrimeStat:A Spatial Statistics Program for the Analysis of Crime 
Incident Locations ( $(v$ 3.I).Washington, D.C.: Ned Levine \& Associates, Houston / The National Institute of Justice.

Moellering, Harold, Henri J.G.L. Aalders y Aaron Crane (2005). World spatial metadata standards. Hardbound: Pergamon, 710 p.

ONU-Hábitat (2006). Safer cities program [en línea]. Disponible en: http://www. unhabitat.org/content.asp

Osborne, Deborah y Susan Wernicke (2003). Introduction to Crime Analysis: Basic Resources for Criminal Justice Practice. Nueva York: Haworth Press.

Pain, Rachel (2000). "Place, social relations and the fear of crime: A review". Progress in Human Geography, 24 (3), 365-387.

PGR (2005). Sistema de Información Estadístico (SIE) [en línea]. México: Procuraduría General de la República. Disponible en: www.pgr.gob. $\mathrm{mx} /$ temas\%20relevantes/estadistica/historico0 I04.asp [2009, I4 de diciembre].

\section{SOPORTETÉCNICO}

ESRI (2005). ArcGIS 9. What is ArcGIS 9. I?, ESRI®, USA, 200I-2005.

MapViewSVG (2007). MapViewSVG 4.2., Freising/Germany. 
